\title{
MHD stagnation point flow of Jeffrey fluid by a radially stretching surface with viscous dissipation and Joule heating
}

\author{
Tasawar Hayat $^{1,2}$, Muhammad Waqas ${ }^{1}$, Sabir Ali Shehzad ${ }^{3 *}$, Ahmed Alsaedi $^{2}$ \\ ${ }^{1}$ Department of Mathematics, Quaid-I-Azam University 45320 Islamabad 44000, Pakistan. \\ ${ }^{2}$ Nonlinear Analysis and Applied Mathematics (NAAM) Research Group, Department of Mathematics, Faculty of Science, King \\ Abdulaziz University, P. O. Box 80257, Jeddah 21589, Saudi Arabia. \\ ${ }^{3}$ Department of Mathematics, Comsats Institute of Information Technology, Sahiwal 57000, Pakistan. \\ * Corresponding author. E-mail: ali_qau70@yahoo.com
}

\begin{abstract}
The steady stagnation-point flow of an electrically conducting fluid due to convectively heated stretched disk in the radial direction is considered. Effects of viscous dissipation and Joule heating are present. Mathematical modelling is based upon constitutive relations of Jeffrey fluid. The governing partial differential equations are first transformed into the coupled system of ordinary differential equations and then solved for the convergent series solutions. Numerical values of skin friction coefficient and local Nusselt number are also computed and analysed.
\end{abstract}

Keywords: MHD stagnation point flow; Jeffrey fluid; Viscous dissipation; Joule heating.

\section{INTRODUCTION}

Understanding and modelling for the flows related to nonNewtonian fluids are of both fundamental and practical significance in the industrial and engineering applications. The rheological characteristics of such fluids are important in the flows of nuclear fuel slurries, lubrication with heavy oils and greases, paper coating, plasma and mercury, fossil fuels, polymers etc. These fluids have a nonlinear relationship between the shear stress and shear rate. The associated equations of non-Newtonian fluids are very complex and higher order than the governing equations of viscous fluid. The nonNewtonian fluids in general are divided into three main classes namely the differential, rate and integral types. The simplest subclass of non-Newtonian fluids is the rate type material. The present study considers the Jeffrey fluid model. This fluid model exhibits the properties of ratio of relaxation to retardation times and retardation time. Few attempts about Jeffrey fluid model are mentioned in the studies by Kothandapani and Srinivas (2008), Tripathi et al. (2011), Hayat et al. (2012), Abbasi et al. (2015) and Farooq et al. (2015).

Fluid motion in the region of a stagnation point exists on all moving solid bodies. The role of stagnation point is important because the separation streamlines passing through them describe different flow regions. Thus the problems studying the stagnation point flow over a stretching surface are classic in fluid mechanics. Initially Hiemenz (1911) studied the steady flow of viscous fluid in the neighborhood of a stagnation point. Later the seminal work of Hiemenz (1911) was extended by the various researchers. For instance Attia (2007) presented the axisymmetric stagnation point flow towards a stretching surface in the presence of uniform magnetic field and heat generation. Zhu et al. (2010) presented an analysis to study the slip condition in stagnation point flow generated by power law stretching sheet. Turkyilmazoglu (2012a) explored the stagnation point flow for three-dimensional steady flow of viscous fluid by a stretchable disk. Soret and Dufour effects in stagnation point flow of Jeffrey fluid are examined by Shehzad et al. (2013).

Boundary layer flow due to stretched surface is a subject of abundant studies at present. It is because of occurrence of such flows in various engineering and industrial processes like cooling of metallic sheets in a bath, annealing and thinning of copper wires, aerodynamic extrusion of plastic and rubber sheets, drawing of plastic films and sheets, glass fiber and paper production etc. (for details see Crane, 1970; Hayat and Awais, 2011; Hayat et al., 2011). Mukhopadhyay (2013) studied magnetohydrodynamic (MHD) boundary layer flow and heat transfer by an exponentially stretching sheet embedded in a thermally stratified medium. Numerical simulation of MHD nanofluid flow and heat transfer with viscous dissipation is reported by Sheikholeslami et al. (2014). Very recently Hayat et al. (2015) examined MHD stagnation-point flow of Jeffrey fluid over a convectively heated stretching sheet.

The purpose of present study is to discuss the MHD stagnation point flow of Jeffrey fluid by a radially stretching sheet. The sheet possesses convective type surface condition. It is noticed that previous literature for heat transfer over a stretching surface is mainly restricted to either prescribed temperature or heat flux at the wall. Heat transfer due to convective boundary conditions has a definite role in processes such as thermal energy storage, gas turbines and nuclear plants etc. (see Aziz, 2009; Hayat et al., 2014a; Makinde and Aziz, 2010). Series solutions of the resulting systems are obtained by homotopy analysis method (HAM) (see Abbasbandy et al., 2013; Hassan and Rashidi, 2014; Hayat et al., 2013; Hayat et al., 2014b; Rashidi et al., 2012a, 2012b; Turkyilmazoglu, 2012b). Relevant convergence criteria are established. Plots for different quantities are shown and discussed.

\section{FORMULATION}

Consider the two-dimensional stagnation point flow of Jeffrey fluid by a radially stretching surface. The surface coincides with the plane $z=0$ whereas the fluid occupies the region $z \geq 0$. A uniform magnetic field of strength $B_{0}$ is applied along the $y$-axis. The velocity distribution (Attia, 2007) in the flow close to the stagnation point is given by $U_{e}(r)=a r, W_{e}(z)=-2 a z$ and the velocity of the stretching sheet is $U_{w}(r)=c r$. Here $a$ and $c$ are the positive constants and $r$ is the radial coordinate. The constitutive relationships of 
Jeffrey fluid are (Hayat et al., 2012; Kothandapani and Srinivas, 2008):

$\mathbf{T}=-p \mathbf{I}+\mathbf{S}$,

$\mathbf{S}=\frac{\mu}{1+\lambda_{1}}\left(\mathbf{A}_{1}+\lambda_{2} \frac{d}{d t} \mathbf{A}_{1}\right)$

where $p$ denotes the pressure, $\mathbf{I}$ the identity tensor, $\mu$ the dynamic viscosity, $\lambda_{1}$ the ratio of relaxation and retardation times and $\lambda_{2}$ the retardation time. The quantities $\mathbf{A}_{1}$ and $\frac{d}{d t} \mathbf{A}_{1}$ are defined by

$$
\begin{aligned}
& \mathbf{A}_{1}=\nabla v+(\nabla v)^{T}, \\
& \frac{d}{d t} \mathbf{A}_{1}=\frac{d}{d t}\left(\mathbf{A}_{1}\right)+(v \cdot \nabla) \mathbf{A}_{1} .
\end{aligned}
$$

Using the equations of continuity and momentum, the resulting boundary layer equations can be expressed as follows:

$$
\frac{\partial u}{\partial r}+\frac{u}{r}+\frac{\partial w}{\partial z}=0
$$

$$
\begin{aligned}
u \frac{\partial u}{\partial r}+w \frac{\partial u}{\partial z}= & \frac{v}{1+\lambda_{1}}\left[\frac{\partial^{2} u}{\partial z^{2}}+\lambda_{2}\left(\begin{array}{c}
u \frac{\partial^{3} u}{\partial r \partial z^{2}}+w \frac{\partial^{3} u}{\partial z^{3}} \\
\left.\left.+\frac{\partial u}{\partial z} \frac{\partial^{2} u}{\partial r \partial z}+\frac{\partial w}{\partial z} \frac{\partial^{2} u}{\partial z^{2}}\right)\right]
\end{array}\right.\right. \\
& -\frac{\sigma B_{0}^{2}}{\rho}\left(u-U_{\infty}\right)+U_{\infty} \frac{d U_{\infty}}{d r}
\end{aligned}
$$

$u \frac{\partial T}{\partial r}+w \frac{\partial T}{\partial z}=\frac{k}{\rho c_{p}}\left(\frac{\partial^{2} T}{\partial z^{2}}\right)$

$+\frac{\mu}{\rho c_{p}}\left(\frac{\partial u}{\partial z}\right)^{2}+\frac{\sigma B_{0}^{2}}{\rho} u$,

subject to the following conditions

$u=U_{w}(r)=c r, w=0$,

$-k \frac{\partial T}{\partial z}=h\left(T_{w}-T\right)$ at $z=0$,

$u \rightarrow U_{\infty}(r)=$ ar,$T \rightarrow T_{\infty}$ as $z \rightarrow \infty$

where $u$ and $w$ are the velocity components along the radial $(r)$ and axial $(z)$ directions, respectively, $T$ is the temperature of fluid, $T_{w}$ is the temperature at the wall, $c_{p}$ is the specific heat, $k$ is the thermal conductivity, $\rho$ is the density, $v$ is the kinematic viscosity, $h$ is the convective heat transfer coefficient and $T_{\infty}$ is the ambient temperature.

Considering the dimensionless variables

$$
\begin{aligned}
& \eta=z \sqrt{\frac{c}{v}}, u=c r f^{\prime}(\eta), w=-2 \sqrt{c v} f(\eta), \\
& \theta(\eta)=\frac{T-T_{\infty}}{T_{w}-T_{\infty}}
\end{aligned}
$$

the continuity equation (1) is identically satisfied and the resulting problems in $f$ and $\theta$ are reduced to the following forms:

$$
\begin{aligned}
& f^{\prime \prime \prime}+\beta\left(f^{\prime \prime 2}-f^{\prime \prime \prime \prime \prime}-2 f f^{\prime \prime \prime \prime}\right)+ \\
& \left(1+\lambda_{1}\right)\left[\begin{array}{c}
H a^{2}\left(A-f^{\prime}\right)+A^{2} \\
+2 f f^{\prime \prime}-f^{\prime 2}
\end{array}\right]=0, \\
& \theta^{\prime \prime}+2 \operatorname{Pr} f \theta^{\prime}+\operatorname{Pr} E c f^{\prime \prime 2}+\operatorname{Pr} E c H a^{2} f^{\prime 2}=0, \\
& f^{\prime}(\eta)=1, f(\eta)=0, \theta^{\prime}=-\gamma[1-\theta(0)] \text { at } \eta=0, \\
& f^{\prime}(\eta)=A, \theta(\eta)=0 \text { as } \eta \rightarrow \infty,
\end{aligned}
$$

in which $\beta$ denotes the Deborah number, $\mathrm{Ha}^{2}$ the Hartmann number, $A$ the ratio of rates of stream velocity to the stretching velocity, $\operatorname{Pr}$ the Prandtl number, $E c$ the Eckert number and $\gamma$ the Biot number. These parameters are defined as follows:

$$
\begin{gathered}
\beta=\lambda_{2} c, H a^{2}=\frac{\sigma B_{0}^{2}}{\rho c}, A=\frac{a}{c}, \operatorname{Pr}=\frac{v}{\alpha}, \\
\alpha=\frac{k}{\rho c_{p}}, \gamma=\frac{h}{k \sqrt{\frac{v}{c}}}, E c=\frac{U_{w}^{2}}{c_{p}\left(T_{w}-T_{\infty}\right)} .
\end{gathered}
$$

Non-dimensional skin friction coefficient and Nusselt number are given by

$$
\begin{aligned}
& \frac{1}{2} C_{f} \sqrt{\operatorname{Re}_{r}}=\frac{1}{1+\lambda_{1}}\left(\begin{array}{l}
f^{\prime \prime}(0)-\beta f(0) \\
f^{\prime \prime \prime}(0)+\beta f^{\prime}(0) f^{\prime \prime}(0)
\end{array}\right), \\
& \left(\operatorname{Re}_{r}\right)^{-1 / 2} N u_{r}=-\theta^{\prime}(0),
\end{aligned}
$$$$
\text { where } \operatorname{Re}_{r}=c r^{2} / v \text { is local Reynolds number. }
$$

\section{SERIES SOLUTIONS}

The initial guesses and auxiliary linear operators are given below:

$$
\begin{aligned}
& f_{0}(\eta)=A \eta+(1-A)\left(1-e^{-\eta}\right), \theta_{0}(\eta)=\frac{\gamma e^{-\eta}}{1+\gamma}, \\
& L_{f}=f^{\prime \prime \prime}-f^{\prime}, \quad L_{\theta}=\theta^{\prime \prime}-\theta .
\end{aligned}
$$

The above auxiliary linear operators satisfy the following properties

$$
L_{f}\left(C_{1}+C_{2} e^{\eta}+C_{3} e^{-\eta}\right)=0, L_{\theta}\left(C_{4} e^{\eta}+C_{5} e^{-\eta}\right)=0,
$$


in which $C_{i}(i=1-5)$ are the arbitrary constants.

The corresponding problems at the zeroth order are given in the following forms:

$$
\begin{aligned}
& (1-p) L_{f}\left[\hat{f}(\eta ; p)-f_{0}(\eta)\right] \\
& =p \hbar_{f} \mathbf{N}_{f}[\hat{f}(\eta ; p), \hat{\theta}(\eta, p)], \\
& (1-p) L_{\theta}\left[\hat{\theta}(\eta ; p)-\theta_{0}(\eta)\right] \\
& =p \hbar_{\theta} \mathbf{N}_{\theta}[\hat{f}(\eta ; p), \hat{\theta}(\eta, p)],
\end{aligned}
$$

$\hat{f}(0 ; p)=0, \hat{f}^{\prime}(0 ; p)=1, \hat{f}^{\prime}(\infty ; p)=A$,

$\hat{\theta}^{\prime}(0, p)=-\gamma(1-\theta(0)), \hat{\theta}(\infty, p)=0$,

$\mathbf{N}_{f}[\hat{f}(\eta, p), \hat{\theta}(\eta, p)]=\frac{\partial^{3} \hat{f}(\eta, p)}{\partial \eta^{3}}$

$+\beta\left(\begin{array}{l}\left(\frac{\partial \hat{f}(\eta, p)}{\partial \eta}\right)^{2}-\frac{\partial \hat{f}(\eta, p)}{\partial \eta} \frac{\partial^{3} \hat{f}(\eta, p)}{\partial \eta^{3}} \\ -2 \hat{f}(\eta, p) \frac{\partial^{4} \hat{f}(\eta, p)}{\partial \eta^{4}}\end{array}\right)$

$+\left(1+\lambda_{1}\right)\left(\left(\begin{array}{c}H a^{2} A-H a^{2} \frac{\partial \hat{f}(\eta, p)}{\partial \eta}+A^{2}+ \\ 2 \hat{f}(\eta, p) \frac{\partial^{2} \hat{f}(\eta, p)}{\partial \eta^{2}}-\left(\frac{\partial \hat{f}(\eta, p)}{\partial \eta}\right)^{2}\end{array}\right)\right)$,

$\mathbf{N}_{\theta}[\hat{\theta}(\eta, p), \hat{f}(\eta, p)]=\frac{\partial^{2} \hat{\theta}(\eta, p)}{\partial \eta^{2}}$

$+2 \operatorname{Pr} \hat{f}(\eta, p) \frac{\partial \hat{\theta}(\eta, p)}{\partial \eta}+\operatorname{Pr} E c\left(\frac{\partial^{2} \hat{f}(\eta, p)}{\partial \eta^{2}}\right)^{2}$

$+\operatorname{Pr} E c H a^{2}\left(\frac{\partial \hat{f}(\eta, p)}{\partial \eta}\right)^{2}$

Here $p$ is an embedding parameter and $\hbar_{f}$ and $\hbar_{\theta}$ are the non-zero auxiliary parameters. The nonlinear operators are represented by $\mathbf{N}_{f}$ and $\mathbf{N}_{\theta}$. When $p=0$ and $p=1$ one has

$\hat{f}(\eta ; 0)=f_{0}(\eta), \hat{\theta}(\eta, 0)=\theta_{0}(\eta)$,

$\hat{f}(\eta ; 1)=f(\eta), \hat{\theta}(\eta, 1)=\theta(\eta)$.

Clearly when $p$ is increased from 0 to 1 then $f(\eta, p)$ and $\theta(\eta, p)$ vary from $f_{0}(\eta), \theta_{0}(\eta)$ to $f(\eta)$ and $\theta(\eta)$. By Taylor's expansion we have

$$
\begin{aligned}
& f(\eta, p)=f_{0}(\eta)+\sum_{m=1}^{\infty} f_{m}(\eta) p^{m}, \\
& f_{m}(\eta)=\left.\frac{1}{m !} \frac{\partial^{m} f(\eta ; p)}{\partial \eta^{m}}\right|_{p=0},
\end{aligned}
$$

$\theta(\eta, p)=\theta_{0}(\eta)+\sum_{m=1}^{\infty} \theta_{m}(\eta) p^{m}$

$\theta_{m}(\eta)=\left.\frac{1}{m !} \frac{\partial^{m} \theta(\eta ; p)}{\partial \eta^{m}}\right|_{p=0}$

The convergence of above series strongly depends upon $\hbar_{f}$ and $\hbar_{\theta}$. Here $\hbar_{f}$ and $\hbar_{\theta}$ are selected properly so that the Eqs. (25) and (26) converge at $p=1$ then we can write

$f(\eta)=f_{0}(\eta)+\sum_{m=1}^{\infty} f_{m}(\eta)$

$\theta(\eta)=\theta_{0}(\eta)+\sum_{m=1}^{\infty} \theta_{m}(\eta)$

The resulting problems at mth order deformation are:

$L_{f}\left[f_{m}(\eta)-\chi_{m} f_{m-1}(\eta)\right]=\hbar_{f} \mathbf{R}_{f}^{m}(\eta)$,

$L_{\theta}\left[\theta_{m}(\eta)-\chi_{m} \theta_{m-1}(\eta)\right]=\hbar_{\theta} \mathbf{R}_{\theta}^{m}(\eta)$,

$f_{m}(0)=f_{m}^{\prime}(0)=f_{m}^{\prime}(\infty)=0$,

$\theta_{m}^{\prime}(0)-\gamma \theta_{m}(0)=\theta_{m}(\infty)=0$,

$\mathbf{R}_{f}^{m}(\eta)=f_{m-1}^{\prime \prime \prime}(\eta)+\beta \sum_{k=0}^{m-1}\left[\begin{array}{l}f_{m-1-k}^{\prime \prime} f_{k}^{\prime \prime}- \\ f_{m-1-k}^{\prime} f_{k}^{\prime \prime \prime}-2 f_{m-1-k} f_{k}^{\prime \prime \prime \prime}\end{array}\right]$

$+2\left(1+\lambda_{1}\right) \sum_{k=0}^{m-1} f_{m-1-k} f_{k}^{\prime \prime}-\left(1+\lambda_{1}\right) \sum_{k=0}^{m-1} f_{m-1-k}^{\prime} f_{k}^{\prime}$

$-\left(1+\lambda_{1}\right) H a^{2} f_{m-1}^{\prime}+\left(1+\lambda_{1}\right) H a^{2} A\left(1-\chi_{m}\right)$

$+\left(1+\lambda_{1}\right) A^{2}\left(1-\chi_{m}\right)$,

$\mathbf{R}_{\theta}^{m}(\eta)=\theta_{m-1}^{\prime \prime}(\eta)+2 \operatorname{Pr} \sum_{k=0}^{m-1} \theta_{m-1-k}^{\prime} f_{k}$

$+\operatorname{Pr} E c \sum_{k=0}^{m-1} f_{m-1-k}^{\prime \prime} f_{k}^{\prime \prime}+\operatorname{Pr} E c H a^{2} \sum_{k=0}^{m-1} f_{m-1-k}^{\prime} f_{k}^{\prime}$,

Solving the above mth order deformation problems, the general solutions can be expressed as follows:

$f_{m}(\eta)=f_{m}^{*}(\eta)+C_{1}+C_{2} e^{\eta}+C_{3} e^{-\eta}$,

$\theta_{m}(\eta)=\theta_{m}^{*}(\eta)+C_{4} e^{\eta}+C_{5} e^{-\eta}$,

where $f_{m}^{*}$ and $\theta_{m}^{*}$ indicate the special solutions. 


\section{Convergence of the homotopy solutions}

It is obvious that the series solutions (27) and (28) consist of the non-zero auxiliary parameters $\hbar_{f}$ and $\hbar_{\theta}$ which can adjust and control the convergence. For the range of admissible values of $\hbar_{f}$ and $\hbar_{\theta}$ of the functions $f^{\prime \prime}(0)$ and $\theta^{\prime}(0)$, the $\hbar_{f}$ and $\hbar_{\theta}$ curves are plotted for 12-th order of approximations. Figs. 1 and 2 depict that the ranges for the admissible values of $\hbar_{f}$ and $\hbar_{\theta}$ are $-1.30 \leq \hbar_{f} \leq-0.20$ and $-1.40 \leq \hbar_{\theta} \leq-0.30$ respectively.

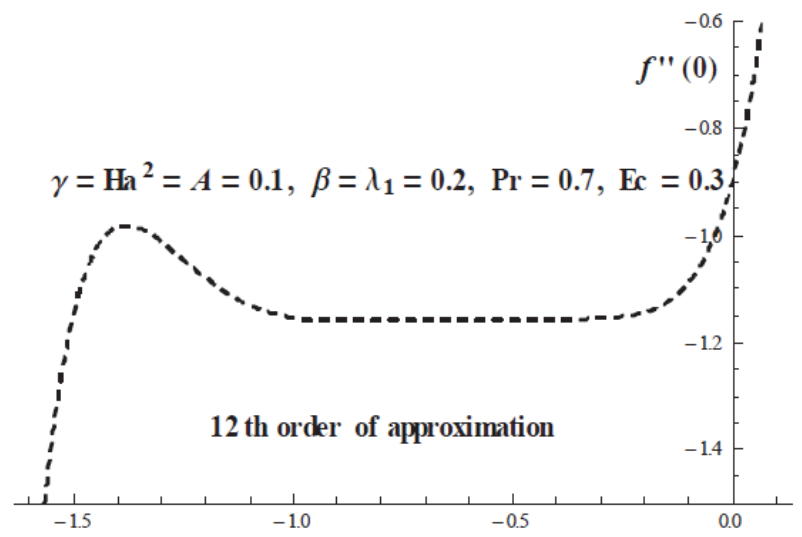

Fig. 1. $\hbar$-curve for the function $f(\eta)$.

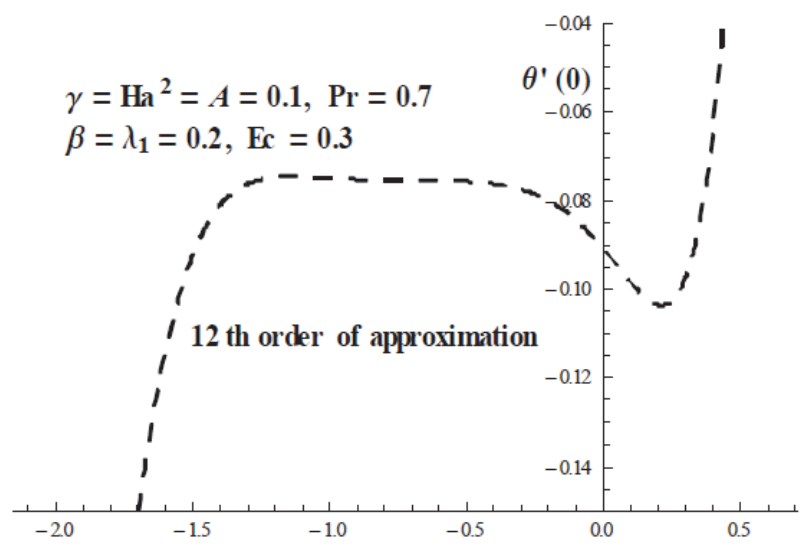

Fig. 2. $\hbar$-curve for the function $\theta(\eta)$.

\section{RESULTS AND DISCUSSION}

The governing dimensionless equations (10) and (11) along with the boundary conditions (12) are solved through homotopic procedure. The computed homotopic solutions are plotted to examine the influences of emerging parameters namely Deborah number $\beta$, Hartmann number $\mathrm{Ha}^{2}$, ratio parameter $A$, Prandtl number Pr, Eckert number $E c$ and Biot number $\gamma$ on the velocity field $f^{\prime}(\eta)$ and temperature profile $\theta(\eta)$. Figs. 3-6 show the influence of dimensionless parameters $A, H a^{2}, \quad \beta$ and $\lambda_{1}$ on the velocity field. The effects of $A$ on the velocity profile $f^{\prime}(\eta)$ are shown in Fig. 3. This Fig. indicates that an increase in $A$ enhances the veloci-

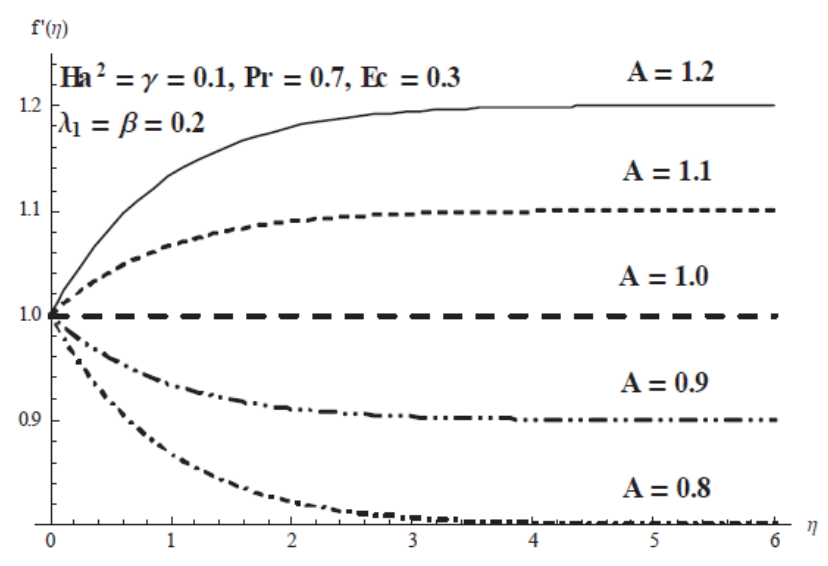

Fig. 3. Effects of $A$ on $f^{\prime}(\eta)$.

ty and boundary layer thickness $(0 \leq A<1)$. It is noted that the boundary layer thickness vanishes when $A=1.0$. For $A>1$, the velocity increases and the boundary layer thickness decreases by increasing $A$. Fig. 4 depicts the influence of $H a^{2}$ on the velocity profile $f^{\prime}(\eta)$. It has been noticed that the magnetic field retards the flow. Physically the apparent viscosity of the fluid increases to the point of becoming a viscoelastic solid when magnetic field is applied to any fluid. It is of great interest that the yield stress of the fluid can be controlled accurately through variation of the magnetic field intensity. The result is that the ability of the fluid to transmit force can be controlled with the help of an electromagnet which gives rise to many possible control-based applications including MHD power generation, electromagnetic casting of metals, MHD ion propulsion etc. Influence of the Deborah number $\beta$ on $f^{\prime}(\eta)$ is shown in Fig. 5. It is observed that the velocity of fluid and associated boundary layer thickness increase for larger $\beta$. Since the Deborah number $\beta$ is dependent upon $\lambda_{2}$ (retardation time) and physically larger retardation time of any material makes it less viscous which ultimate results in an increase of its motion. Fig. 6 shows the influence of $\lambda_{1}$ on $f^{\prime}(\eta)$. It is seen that an increase in $\lambda_{1}$ retards the flow.

Here increase in $\lambda_{1}$ enhances the relaxation time i.e., more time is required for a perturbed system to retain its original position. The drag forces increase and consequently the reduction occurs in the velocity.

Figs. 7-9 show the influence of different parameters $E c, \gamma$ and $\operatorname{Pr}$ on temperature profile $\theta(\eta)$. Characteristics of Eckert number $E c$ on temperature profile are sketched in Fig. 7. It is found that the temperature and thermal boundary layer thickness are enhanced with an increase in Ec. With an increase in $E c$ the heat energy is stored in the fluid due to friction forces which enhances the temperature profile. Influence of Biot number $\gamma$ on temperature profile is shown in Fig. 8. It is noticed that the stronger convective heating at the sheet allows the thermal effect to penetrate deeper into the quiescent fluid. This results in the larger temperature and thicker thermal boundary layer. Increasing values of Prandtl number Pr decrease conduction and enhance pure convection i.e. transfer of heat through unit area. That is why the temperature and thermal boundary layer thickness decrease with an increase in $\operatorname{Pr}$ (see Fig. 9). 


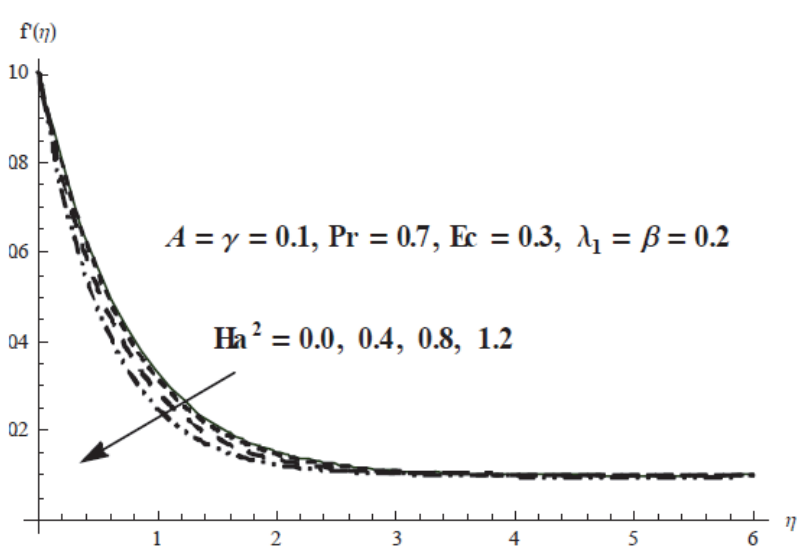

Fig. 4. Effects of $H a^{2}$ on $f^{\prime}(\eta)$.

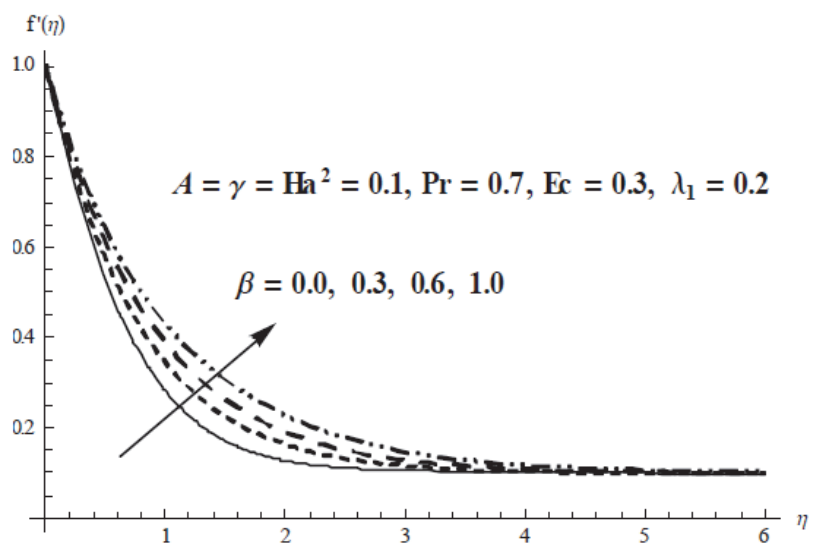

Fig. 5. Effects of $\beta$ on $f^{\prime}(\eta)$.

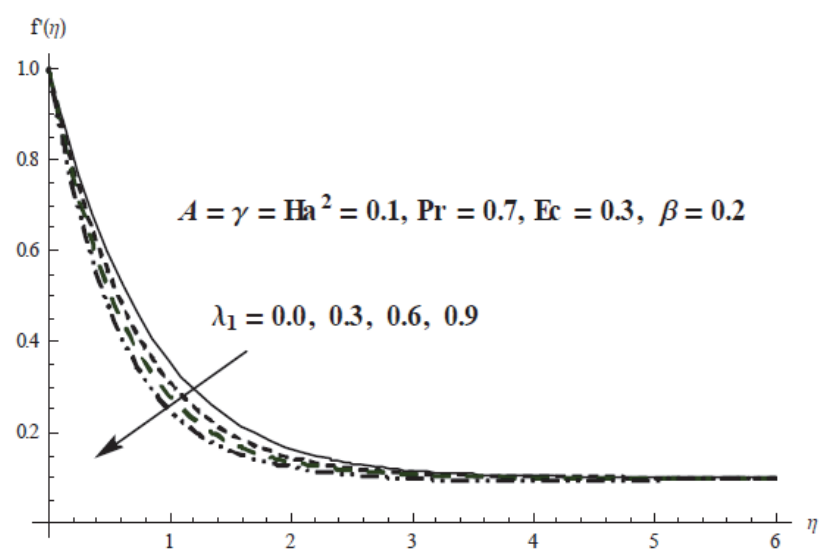

Fig. 6. Effects of $\lambda_{1}$ on $f^{\prime}(\eta)$.

Figs. 10-12 show the variations of ratio of rates of free stream velocity to the stretching velocity $A$, Hartmann number $\mathrm{Ha}^{2}$, ratio of relaxation and retardation times $\lambda_{1}$, Deborah number $\beta$, Biot number $\gamma$ and Eckert number $E_{c}$ on skin friction coefficient and local Nusselt number respectively.

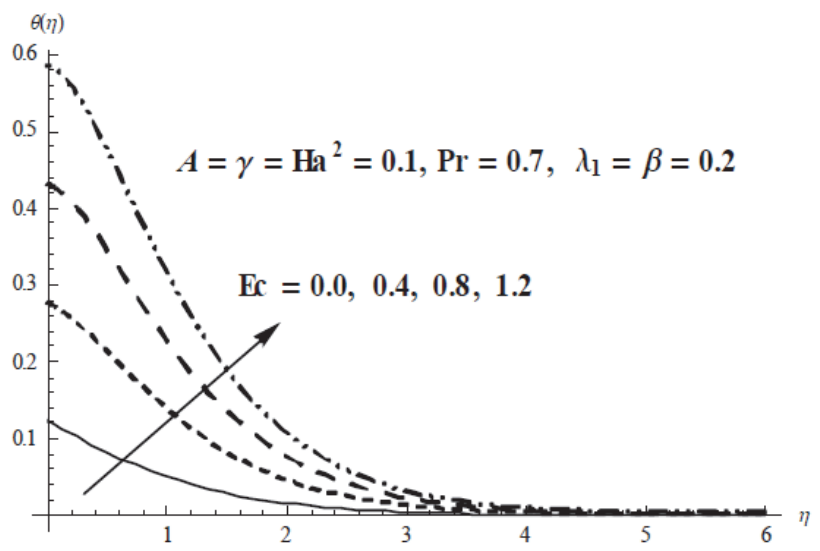

Fig. 7. Effects of $E c$ on $\theta(\eta)$.

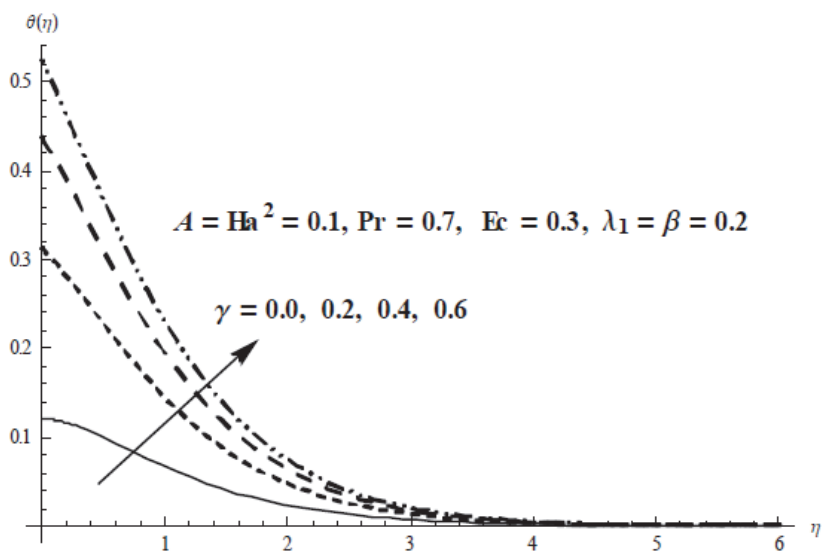

Fig. 8. Effects of $\gamma$ on $\theta(\eta)$.

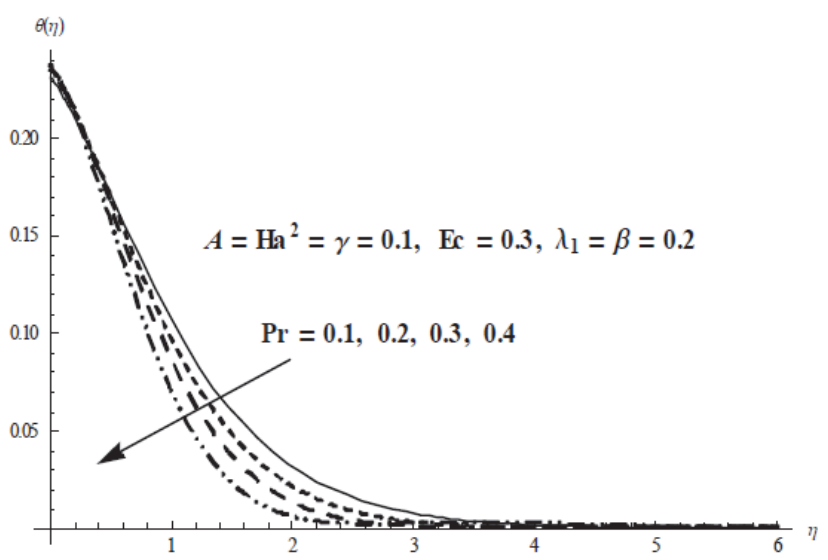

Fig. 9. Effects of $\operatorname{Pr}$ on $\theta(\eta)$.

Figs. 10 and 11 are drawn to examine the variations of $A$ and $\lambda_{1}$ on skin friction coefficient. It is found that skin friction coefficient enhances for larger values of $A$ and $\lambda_{1}$. Moreover, the local Nusselt number which represents the heat transfer rate at the surface increases for larger $\gamma$ and $E c$ (see Fig. 12). 


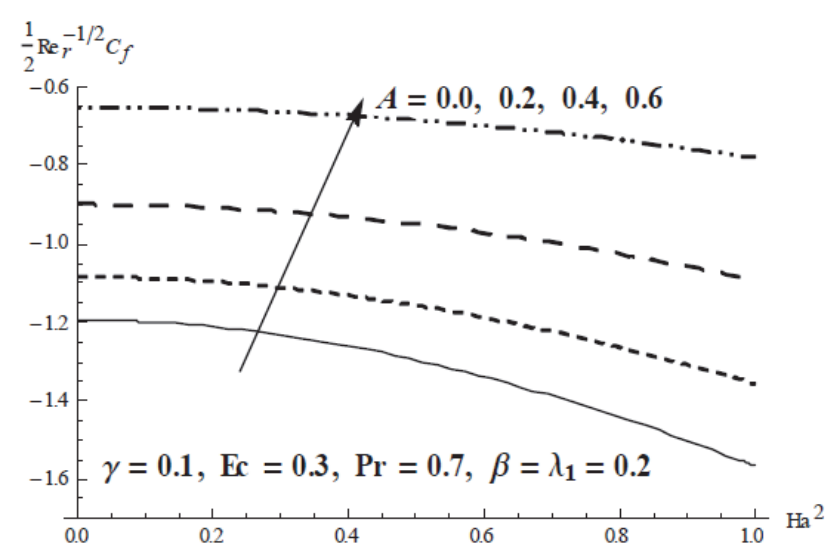

Fig. 10. Effects of $A$ and $H a^{2}$ on skin-friction coefficient.

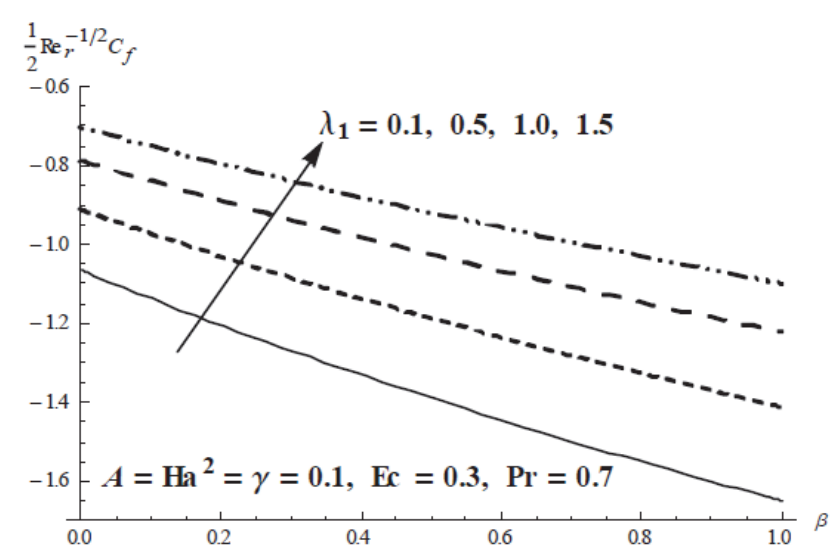

Fig. 11. Effects of $A$ and $\lambda_{I}$ and $\beta$ on skin-friction coefficient.

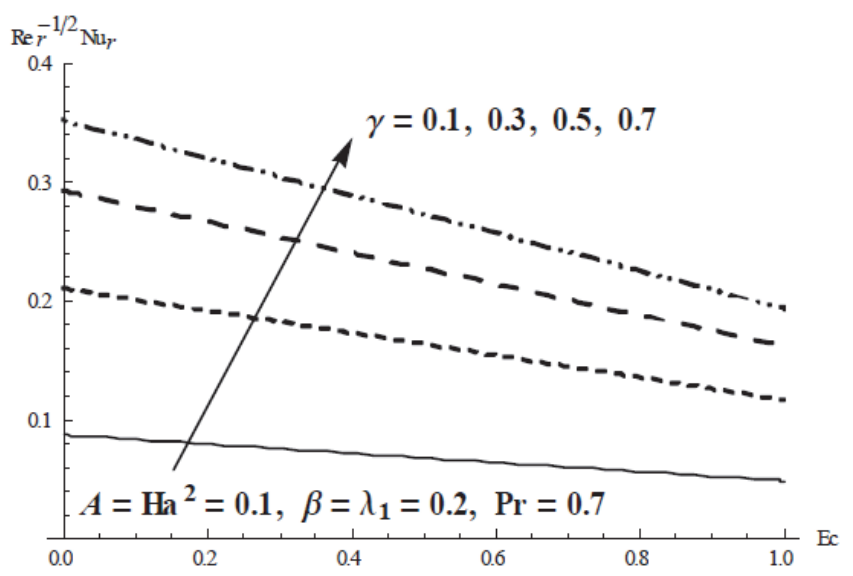

Fig. 12. Effects of $\gamma$ and $E c$ on local Nusselt number.

Table 1 is presented to find how much order of approximations is necessary for a convergent solution. It is noticed that 15 th order of approximations are sufficient for the velocity field whereas 25 th order of approximation are required for the temperature field. Table 2 is presented for the numerical values of skin friction coefficient for different values of $A$, $\mathrm{Ha}^{2}, \quad \beta$ and $\lambda_{1}$. It is noticed that the skin friction coefficient increases for larger values of $H a^{2}$ and $\beta$. However the skin friction coefficient are decreased when $A$ and $\beta$ increase.
Tables 2 and 3 are presented for the numerical values of local Nusselt number for different values of $\gamma, E c$ and Pr. This Table represents that the values of heat transfer rate become larger when we increase the values of $\gamma$ and $\operatorname{Pr}$ while it is smaller with an increase in $E c$.

Table 1. Convergence of homotopy solutions when $\lambda_{1}=0.2$, $A=0.1, \quad \operatorname{Pr}=0.7, \gamma=0.1, \quad E c=0.3, \quad \beta=0.2$ and $H a^{2}=0.1$.

\begin{tabular}{ccc}
\hline Order of approximation & $-f^{\prime \prime}(0)$ & $-\theta^{\prime}(0)$ \\
\hline 1 & 1.0557 & 0.087226 \\
5 & 1.1553 & 0.078061 \\
10 & 1.1570 & 0.075656 \\
15 & 1.1570 & 0.075298 \\
20 & 1.1570 & 0.075218 \\
25 & 1.1570 & 0.075169 \\
30 & 1.1570 & 0.075169 \\
35 & 1.1570 & 0.075169 \\
\hline
\end{tabular}

Table 2. Values of skin-friction coefficient for different values of the parameters $A, \mathrm{Ha}^{2}, \beta$ and $\lambda_{1}$ when $\operatorname{Pr}=0.7, \gamma=0.1$ and $E c=0.3$.

\begin{tabular}{lllll}
\hline$A$ & $H a^{2}$ & $\beta$ & $\lambda_{1}$ & $\frac{1}{2} C_{f} \sqrt{\mathrm{Re}_{r}}$ \\
\hline 0.0 & 0.1 & 0.2 & 0.2 & 1.2017 \\
0.1 & & & & 1.1570 \\
0.2 & & & & 1.0920 \\
0.1 & 0.0 & & & 1.1534 \\
& 0.3 & & & 1.1856 \\
& 0.5 & & & 1.2412 \\
& 0.1 & 0.0 & & 1.0298 \\
& & 0.1 & & 1.0946 \\
& & 0.3 & & 1.2170 \\
& & 0.2 & 0.0 & 1.2674 \\
& & & 0.2 & 1.1570 \\
& & & 0.4 & 1.0711 \\
\hline
\end{tabular}

Table 3. Values of local Nusselt number for different values of $\gamma$, $E c$ and $\operatorname{Pr}$ when $A=H a^{2}=0.1$ and $\beta=\lambda_{1}=0.2$.

\begin{tabular}{llll}
\hline$\gamma$ & $E c$ & $\operatorname{Pr}$ & $-\theta^{\prime}(0)$ \\
\hline 0.5 & 0.3 & 0.7 & 0.2236 \\
0.7 & & & 0.2688 \\
0.9 & & & 0.3020 \\
0.9 & 0.0 & & 0.3953 \\
& 0.5 & & 0.3023 \\
& 1.0 & & 0.2087 \\
0.9 & 1.0 & 0.5 & 0.2730 \\
& & 0.6 & 0.2889 \\
& & 0.8 & 0.3381 \\
\hline
\end{tabular}




\section{CONCLUDING REMARKS}

Axisymmetric stagnation point flow of Jeffrey fluid is explored. The flow is induced by a radially stretching surface. The concept of convective boundary condition is analyzed. The final outcomes of this investigation are listed below:

- Magnetic field retards the fluid velocity and momentum boundary layer thickness.

- Velocity of fluid can be boosted by increasing its retarding time.

- $\quad$ Prandtl number Pr reduces the temperature field and decreases the thermal boundary layer thickness.

- Both the temperature and thermal boundary layer thickness are enhanced for the larger Biot number $\gamma$.

- Temperature profile increases for larger Eckert number $E c$.

- The values of skin-friction are smaller in case of hydrodynamic flow when we compare it with the hydromagnetic flow.

- The values of local Nusselt number are reduced for larger Eckert number $E c$. For $E c=0$ the results in absence of viscous dissipation can be recovered.

Acknowledgement. We are grateful to the reviewer for his/her useful comments and suggestions to improve the quality of manuscript.

\section{REFERENCES}

Abbasbandy, S., Hashemi, M.S., Hashim, I., 2013. On convergence of homotopy analysis method and its application to fractional integro-differential equations. Quaestiones Mathematicae, 36, 93-105.

Abbasi, F.M., Shehzad, S.A., Hayat, T., Alsaedi, A., Obid, M.A., 2015. Influence of heat and mass flux conditions in hydromagnetic flow of Jeffrey nanofluid. AIP Advances, 5, 037111.

Attia, H.A., 2007. Axisymmetric stagnation point flow towards a stretching surface in the presence of a uniform magnetic field with heat generation. Tamkang J. Sci. Eng., 10, 11-16.

Aziz, A., 2009. A similarity solution for laminar thermal boundary layer over a flat plate with a convective surface boundary condition. Commun. Nonlinear Sci. Numer. Simulat., 14, 1064-1068.

Crane, L.J., 1970. Flow past a stretching plate. Z. Angew. Math. Phys., 21, 645-647.

Farooq, M., Gull, N., Hayat, T., Alsaedi, A., 2015. MHD flow of Jeffrey fluid with Newtonian heating. J. Mech., 1-11, doi: 10.1017/jmech.2014.93.

Hassan, H.N., Rashidi, M.M., 2014. An analytic solution of micropolar flow in a porous channel with mass injection using homotopy analysis method. Int. J. Numer. Methods Heat Fluid Flow, 24, 419-437.

Hayat, T., Awais, M., 2011. Simultaneous effects of heat and mass transfer on time-dependent flow over a stretching surface. Int. J. Numer. Methods Fluids, 67, 1341-1357.

Hayat, T., Mustafa, M., Obaidat, S., 2011. Soret and Dufour effects on the stagnation-point flow of micropolar fluid toward a stretching sheet. ASME J. Fluid Eng., 133, 1-9.

Hayat, T., Awais, M., Alsaedi, A., 2012. Newtonian heating and magnetohydrodynamic effects in flow of a Jeffrey fluid over a radially stretching. Int. J. Physical Sci., 7, 2838-2844.
Hayat, T., Waqas, M., Shehzad, S.A., Alsaedi, A., 2013. Mixed convection radiative flow of Maxwell fluid near a stagnation point with convective condition. J. Mech., 29, 403-409.

Hayat, T., Waqas, M., Shehzad, S.A., Alsaedi, A., 2014a. Effects of Joule heating and thermophoresis on stretched flow with convective boundary conditions. Scientia Iranica B, 21, 682-692.

Hayat, T., Shehzad, S.A., Al-Mezel, S., Alsaedi, A., 2014 b. Three-dimensional flow of an Oldroyd-B fluid over a bidirectional stretching surface with prescribed surface temperature and prescribed surface heat flux. J. Hydrol. Hydromech., 62, 117-125.

Hayat, T., Asad, S., Mustafa, M., Alsaedi, A., 2015. MHD stagnation-point flow of Jeffrey fluid over a convectively heated stretching sheet. Comput. Fluids, 108, 179-185.

Hiemenz, K., 1911. Die Grenzschicht an einem in den gleichförmigen Flüssigkeitsstrom eingetauchten geraden Kreiszylinder. Int. J. Dingler's Polytech., 326, 321-324.

Kothandapani, M., Srinivas, S., 2008. Peristaltic transport of a Jeffrey fluid under the effect of magnetic field in an asymmetric channel. Int. J. Non-Lin. Mech., 43, 915-924.

Makinde, O.D., Aziz, A., 2010. MHD Mixed convection from a vertical plate embedded in a porous medium with a convective boundary condition. Int. J. Therm. Sci., 49, $1813-1820$

Mukhopadhyay, S., 2013. MHD boundary layer flow and heat transfer over an exponentially stretching sheet embedded in a thermally stratified medium. Alexandria Eng. J., 52, 259265.

Rashidi, M.M., Rajvanshi, S.C., Keimanesh, M., 2012a. Study of pulsatile flow in a porous annulus with the homotopy analysis method. Int. J. Numer. Methods Heat Fluid Flow, 22, 971-989.

Rashidi, M.M., Pour, S.A.M., Hayat, T., Obaidat, S., 2012 b. Analytic approximate solutions for steady flow over a rotating disk in porous medium with heat transfer by homotopy analysis method. Comput. Fluids, 54, 1-9.

Shehzad, S.A., Alsaadi, F.E., Monaquel, S.J., Hayat, T., 2013. Soret and Dufour effects on the stagnation point flow of Jeffrey fluid with convective boundary condition. Eur. Phys. J. Plus, 128, 56.

Sheikholeslami, M., Abelman, S., Ganji, D.D., 2014. Numerical simulation of MHD nanofluid flow and heat transfer considering viscous dissipation. Int. J. Heat Mass Transfer, $79,212-222$.

Tripathi, D., Ali, N., Hayat, T., Chaube, M.K., Hendi, A.A., 2011. Peristaltic flow of MHD Jeffrey fluid through a finite length cylindrical tube. Appl. Math. Mech. Engl. Edit., 32, $1148-1160$.

Turkyilmazoglu, M., 2012a. Three dimensional MHD stagnation flow due to a stretchable rotating disk. Int. J. Heat Mass Transfer, 55, 6959-6965.

Turkyilmazoglu, M., 2012b. Solution of Thomas-Fermi equation with a convergent approach. Commun. Nonlinear Sci. Numer. Simulat., 17, 4097-4103.

Zhu, J., Zheng, L., Zhang, Z., 2010. Effects of slip condition on MHD stagnation-point flow over a power-law stretching sheet. Appl. Math. Mech. Engl. Ed., 31, 439-448.

Received 11 May 2015 Accepted 21 July 2015 